\title{
Anesthetic Management of an Adolescent With Marfan Syndrome During Anterior Spinal Fusion
}

\author{
Yoshikazu Yamaguchia, e, Walter Samora ${ }^{\mathrm{b}}$, Jan E. Klamar ${ }^{\mathrm{b}}$, \\ Dmitry Tumin ${ }^{\text {a, c }}$, Joseph D. Tobias ${ }^{\text {a, d }}$
}

\begin{abstract}
Marfan syndrome (MFS) is an autosomal dominant disorder resulting from a mutation of the gene encoding fibrillin-1 (FBN1) on chromosome 15. Fibrillin-1 is a matrix glycoprotein essential for the formation of microfibrils that regulate the formation and repair of connective tissue throughout the body. This primary cellular defect results in a multisystem disorder of connective tissue. Given the multisystem involvement of MFS, anesthetic care is frequently required during surgical or orthopedic procedures. We present a 15-year-old adolescent who required anesthetic care during spinal fusion. The perioperative implications of MFS are reviewed and options for anesthetic care presented.
\end{abstract}

Keywords: Spinal fusion; Kyphoscoliosis; Marfan syndrome; Anticoagulation

\section{Introduction}

Marfan syndrome (MFS) is a systemic disorder resulting from a primary disorder of connective tissue, first described by the Parisian pediatrician, Antonie-Bernard Marfan in 1896 [1, 2]. Its incidence is approximately 1 in 10,000 - 20,000 individuals, and it is inherited as an autosomal dominant trait, although at least $25 \%$ of cases result from a new mutation in the FBN1 gene (see below). The clinical diagnosis is based on the revised Ghent criteria and can be confirmed by genetic testing [3]. The

Manuscript submitted October 3, 2018, accepted October 9, 2018

aDepartment of Anesthesiology and Pain Medicine, Nationwide Children's Hospital, Columbus, OH, USA

bepartment of Orthopedic Surgery, Nationwide Children's Hospital and The Ohio State University, Columbus, OH, USA

'Department of Pediatrics, Nationwide Children's Hospital and The Ohio State University, Columbus, OH, USA

${ }^{\mathrm{d} D e p a r t m e n t}$ of Anesthesiology, The Ohio State University, Columbus, $\mathrm{OH}$, USA

${ }^{\text {e}}$ Corresponding Author: Yoshikazu Yamaguchi, Department of Anesthesiology and Pain Medicine, Nationwide Children's Hospital, 700 Children's Drive, Columbus, OH 43205, USA.

Email: Yoshikazu.Yamaguchi@nationwidechildrens.org

doi: https://doi.org/10.14740/jmc3181 primary organ systems involved include the heart, aorta, eye, musculoskeletal, skin, lung, and central nervous system [3]. Involvement of the heart and aorta are recognized as the major cause of morbidity and mortality in patients with MFS. Dilation of the ascending aorta occurs in $77 \%$ of affected individuals, with progression to dissection in $14 \%$ of patients [4].

The primary cellular defect of MFS is a mutation of the gene encoding fibrillin-1 (FBN1) on chromosome 15 (15q21.1) [5]. Fibrillin-1 is a matrix glycoprotein essential for the formation of microfibrils that regulate the formation and repair of connective tissue throughout the body. These microfibrils are also integral to the normal control and function of growth factors including tissue growth factor-beta (TGF- $\beta$ ), which are responsible for the development and repair of connective tissue. Fibrillin-1 regulates TGF- $\beta$ which activates metalloproteinases (MMPs). The combination of structural microfibril matrix abnormalities and dysregulation of matrix homeostasis mediated by excess TGF- $\beta$ and overexpression of MMPs is responsible for the phenotypic features of MFS in various organ systems $[6,7]$.

The imbalance between MMPs and tissue inhibitors of MMPs (TIMPs) damages the elastin structure, leading to loss of vascular smooth muscle cells, with the associated clinical manifestations involving the aorta and heart. Given the multisystem involvement of MFS, anesthetic care is frequently required during surgical or orthopedic procedures [8]. We present a 15-year-old adolescent who required anesthetic care during spinal fusion. The perioperative implications of MFS are presented, previous reports of anesthetic care reviewed, and options for perioperative care discussed.

\section{Case Report}

Institutional Review Board approval is not required at Nationwide Children's Hospital (Columbus, Ohio) for the presentation of single case reports. The patient was a 15 -year-old, 54 $\mathrm{kg}$ adolescent who presented for posterior spinal fusion for the treatment of thoracolumbar kyphoscoliosis. Her past medical history was significant for MFS, psychiatric disorder with a history of aggressive behavior, and attention deficit disorder. Her past surgical history included mechanical aortic valve replacement 3 years prior to the current surgery. Aortic valve sparing aortic root replacement and mitral valve repair were performed 6 years prior to surgery. Her medication regimen included warfarin $5 \mathrm{mg}$ once a day, aspirin $81 \mathrm{mg}$ once a day 
and losartan $25 \mathrm{mg}$ once a day.

Warfarin and aspirin were discontinued 4 days prior to surgery and full anticoagulation provided by a continuous heparin infusion. Echocardiogram 5 months prior to the procedure demonstrated mitral valve prolapse with mild regurgitation and normal systolic function. The heparin infusion rate was based on laboratory parameters (partial thromboplastin time (PTT) and anti-factor Xa assay). The patient was held nil per $o s$ for $8 \mathrm{~h}$. The heparin infusion was discontinued $4 \mathrm{~h}$ prior to the surgery; however, the PTT remained elevated at $60 \mathrm{~s}$, so the surgical procedure was delayed for 1 hour and a repeat PTT was obtained (44s).

The patient was premedicated with intravenous midazolam $(2 \mathrm{mg})$ through a pre-existing peripheral intravenous cannula, and transported to the operating room, where routine American Society of Anesthesiologists' monitors were placed. Following preoxygenation with $100 \%$ oxygen, anesthesia was induced with propofol $(130 \mathrm{mg})$, lidocaine $(60 \mathrm{mg})$, and sufentanil $(20 \mu \mathrm{g})$. Bag-valve-mask ventilation was provided without difficulty. Rocuronium $(50 \mathrm{mg}$ ) was administered to facilitate endotracheal intubation. Direct laryngoscopy was performed with a Miller 2 blade and revealed a CormackLehane Grade 1 view. A $7.0 \mathrm{~mm}$ cuffed endotracheal tube was placed on the first attempt. Two 16 gauge intravenous cannulas were placed. A 20 gauge arterial cannula was placed in the left radial artery. Neurophysiological monitoring, including motor evoked potentials (MEP) and somatosensory evoked potentials (SSEPs) was performed.

Following our usual practice to allow for neurophysiological monitoring during spinal surgery, anesthesia was maintained with desflurane titrated to maintain the bispectral index (BIS) at 50 - 60 and a sufentanil infusion of $0.1-0.3 \mu \mathrm{g} / \mathrm{kg} / \mathrm{h}$ to maintain the mean arterial pressure at 55 - $65 \mathrm{~mm} \mathrm{Hg}$ [9]. An esmolol infusion $(25-30 \mu \mathrm{g} / \mathrm{kg} / \mathrm{min})$ was administered to maintain the heart rate at the baseline rate of 60 - 80 beats/ min and a lidocaine infusion $(30 \mu \mathrm{g} / \mathrm{kg} / \mathrm{min})$ was administered to prevent arrhythmias and to provide analgesia. The patient was placed in the right lateral decubitus position. The surgical procedure included an anterior arthrodesis of the thoracic and lumbar spine (T11-L4) through an anterior thoracoabdominal incision. The surgical duration was $400 \mathrm{~min}$. Total blood loss was estimated at $750 \mathrm{~mL}$ and urine output was $325 \mathrm{~mL}$. Total fluids included 1,774 $\mathrm{mL}$ of isotonic crystalloid, $500 \mathrm{~mL}$ of $5 \%$ albumin, and one unit of packed red blood cells.

During wound closure, the sufentanil infusion was discontinued. Acetaminophen $(1,000 \mathrm{mg})$ and hydromorphone $(0.5$ $\mathrm{mg}$ ) were administered to provide postoperative analgesia. After completion of the surgical procedure and prior to tracheal extubation, bilateral ultrasound-guided, erector spinae blocks were performed with $0.25 \%$ ropivacaine $(20 \mathrm{~mL})$ with epinephrine (1:200,000) and preservative-free dexamethasone (4 mg). Following completion of the blocks, the patient was turned supine and her trachea was extubated in the operating room. She was transported to the cardiothoracic intensive care unit (CTICU). Postoperative pain control in the CTICU was provided with hydromorphone delivered via a patient controlled analgesia device. Warfarin was restarted on postoperative day (POD) 1. Enoxaparin was administered from POD 1 to 4 . The remainder of her postoperative course was uncompli- cated and she was discharged on POD 6.

\section{Discussion}

MFS is a well described, autosomal dominant connective tissue disease caused by mutation of the FBN1 gene. The diagnosed is based on the revised Ghent nosology established in 2010 [3]. Aortic diameter at the sinuses of Valsalva ( $z$ value $\geq 2$ ), aortic root dissection, ectopia lentis, and the FBN1 mutation are the most important factors for diagnosis [3]. Effective perioperative care begins with a thorough preoperative evaluation, with identification of anatomical and end-organ effects of MFS, as well as adverse effects or implications related to therapeutic interventions, such as the long-term anticoagulation required in our patient. Of primary concern to anesthesia providers is the potential for difficult endotracheal intubation related to a high arched palate and temporomandibular joint (TMJ) dislocation [10-12]. A significant percentage of patients with MFS have a high arched palate (59\%). TMJ involvement is also common, with symptoms of TMJ dysfunction noted in $52 \%$ of patients with MFS, of whom $35 \%$ had previously received medical treatment, and $24 \%$ had symptoms of subluxation [4, 11]. Given these concerns, the appropriate equipment for dealing with a difficult airway, including an indirect laryngoscopy device, should be readily available prior to anesthetic induction [13, 14]. If there are concerns, general anesthesia can be induced by the incremental inhalation of sevoflurane in $100 \%$ oxygen with the maintenance of spontaneous ventilation until effective bagvalve-mask ventilation is demonstrated. Alternatively, as in our patient, intravenous induction can be achieved with propofol, and effective bag-valve-mask ventilation can be followed by the administration of a neuromuscular blocking agent.

Airway involvement with craniofacial abnormalities and laxity of upper airway musculature results in a high prevalence of obstructive sleep apnea (OSA) in patients with MFS, even in the absence of obesity. Craniofacial and upper airway skeletal muscle involvement results in increased nasal airway resistance and upper airway collapse $[15,16]$. Given the residual effects of opioids and neuromuscular blocking agents on respiratory function, postoperative monitoring may be indicated, especially when ongoing pain management requires the use of opioids [17].

In addition to airway abnormalities, respiratory and pulmonary involvement may predispose to barotrauma due to blebs or bullae. The frequency of spontaneous pneumothorax has been reported to be as high as $4-15 \%$ in patients with MFS $[2,18]$. Apical blebs or bullae significantly increase the risk of spontaneous pneumothorax. When there are concerns noted on a preoperative chest radiograph, computed tomography may help in risk stratification. Additionally, chest wall and vertebral bony abnormalities (scoliosis or pectus excavatum) may impact respiratory function. Progressive scoliosis is a wellknown cause of pulmonary dysfunction. Scoliosis with a curve greater than 65, 100, and 120 degrees may result in restrictive lung disease noted only on pulmonary function testing, symptomatic lung disease with a clinical impact, and alveolar hypoventilation, respectively $[19,20]$. 
Patients with MFS and scoliosis or pectus excavatum may have marked reductions in total lung capacity, forced vital capacity, and forced expiratory volume over $1 \mathrm{~s}\left(\mathrm{FEV}_{1}\right)$. Pulmonary function values calculated by body height underestimate true values in patients with MFS because of longbone overgrowth. Expected values calculated by normalized or sitting height are suggested to give a better evaluation of true pulmonary function [21]. Given these concerns, intraoperative strategies to minimize barotrauma may be indicated, including the use of lower tidal volumes, careful monitoring or peak inflating pressure, prolongation of the inspiratory time, and pressure-controlled ventilation with monitoring of exhaled tidal volume. If alterations in compliance or resistance are noted, the potential for a pneumothorax as the etiology must be considered.

Postoperatively, severe kyphoscoliosis related to neuromuscular disease, as well as OSA and primary respiratory involvement, may predispose to respiratory insufficiency or failure. Patients who have abnormal results on their pulmonary function test, particularly a forced vital capacity less than $30 \%$, or those who have hypercapnia preoperatively have been shown to have a higher need for postoperative ventilation [22]. Preoperative optimization of pulmonary function is suggested, including the aggressive treatment of respiratory infections and instructions regarding the use of postoperative incentive spirometry. Non-invasive techniques of respiratory support, such as bilevel positive airway pressure (BiPAP), may facilitate postoperative tracheal extubation and avoid postoperative respiratory insufficiency or failure in patients with pre-existing pulmonary dysfunction $[23,24]$.

As noted in our patient, the cardiovascular system is frequently affected in patients with MFS, including aortic and pulmonary artery dilation, valvular regurgitation, left ventricular dysfunction, and arrhythmias. MVP is the most common cause of severe mitral valve regurgitation, as upregulation of TGF- $\beta$ results in myxomatous changes of the atrioventricular valves [25]. This results in elongation and thickening of atrioventricular valve leaflets. By 30 years of age, the Weibull cumulative distribution has been reported to be $42.6 \%$ for MVP, $56.5 \%$ for MVR of any degree, $6.7 \%$ for severe MVR, and $0.9 \%$ for MV endocarditis in patients with MFS [26]. In children with early onset of severe MFS, mitral valve dysfunction, which can lead to pulmonary hypertension and congestive heart failure, is the leading cause of death in infancy. Tricuspid valve regurgitation and prolapse can also occur, although surgery is rarely required [27]. Aortic valve regurgitation can occur as well, but it generally occurs in the second and third decade of life, related to progressive aortic root dilation.

Aortic root dilation is the most common cardiovascular manifestation of MFS, occurring in $60 \%$ of affected individuals. Aortic root rupture and dissection remain the most lifethreatening events in patients with MFS. The aorta dilates most between 6 to 14 years of age [28]. Management may include both medical and surgical management options. Medical therapy includes a combination of $\beta$-adrenergic antagonists and angiotensin receptor blockers (ARB). Beta-adrenergic blockade reduces heart rate, blood pressure, and aortic root dilation [29]. The target heart rate is less than 60 - 70 beats per minutes at rest [30]. Intraoperatively, esmolol may be used to provide rapid and precise heart rate control. ARBs regulate excessive TGF- $\beta$ signaling, which may play an etiologic role in aortic root dilation [31].

Ascending aorta root replacement is the only way to prevent fatal events including dissection and rupture. Elective surgery is recommended when the maximum aortic root diameter is greater than $5.0-5.5 \mathrm{~cm}$ in adult patients. If the patients have a family history of aortic rupture, or increase in aortic diameter exceeding $1 \mathrm{~cm} /$ year, earlier surgical intervention is recommended. Various surgical procedures have been offered, including various valve sparing procedures which preserve the native aortic valve and eliminate the need for long-term anticoagulation [32-34]. When mechanical valve replacement is performed, long-term anticoagulation with coumadin is required.

Guidelines for perioperative anticoagulation therapy have been published by the American Heart Association and the American College of Cardiology [35]. As in our patient, coumadin or other oral anticoagulation agents are stopped 3 - 4 days prior to surgery, and full anticoagulation started with heparin (unfractionated or low molecular weight) once the international normalized ratio (INR) falls below the therapeutic threshold. Intravenous unfractionated heparin is stopped 4 - 6 $\mathrm{h}$ prior to surgery and subcutaneous low molecular heparin is stopped $12 \mathrm{~h}$ prior to surgery, to allow return of coagulation function to normal. Anticoagulation is restarted as soon as the postoperative bleeding risk allows, typically 12 to $24 \mathrm{~h}$ after surgery.

In addition to valvular dysfunction and aortic root dilation, ventricular arrhythmias may occur in up to $21 \%$ of patients with MFS, with one report suggesting that this was the cause of death in $4 \%$ of patients [36]. Non-sustained ventricular tachycardia was also noted in $40 \%$ of patients. Microfibril abnormalities in the matrix of the myocardium result in arrhythmogenesis and may affect conduction [37]. Given these concerns, a preoperative 12-lead ECG and echocardiogram are suggested. A more thorough workup such as a Holter monitor may be indicated in patients with symptoms.

Ocular involvement, including ectopia lentis, is common in MFS and may predispose to perioperative visual loss (POVL) during spine surgery. Additional ocular involvement may include thinning of the cornea, early onset of cataracts, glaucoma, strabismus, and retinal detachment. Although POVL is rare, its impact on the quality of life is significant, and spinal surgery represents a high risk population with an incidence of POVL ranging from $0.017 \%$ to $0.1 \%$ [38]. Ischemic injury to the optic nerve due to venous congestion is the most common cause of POVL following spinal surgery. Symptoms present immediately after the surgery and are typically bilateral. Risk factors include anemia, hypotension, blood loss, prolonged surgical times, large fluid requirements, male gender and obesity $[39,40]$. Prevention should remain the primary focus, with routine position checks to ensure that there is no direct pressure on the eyes or orbits. Others have suggested the use of colloid instead of crystalloid as well as avoidance of hypotension, anemia, and hypovolemia. A staged procedure should be considered if a long surgery is anticipated.

Finally, intraoperative monitoring of spinal cord function with somatosensory-evoked potentials (SSEPs) and motorevoked potentials (MEPs) further impacts anesthetic manage- 
ment during PSF [41]. Both inhaled and intravenous anesthetic agents may depress SSEP and MEP signal attainment [42]. Our general practice includes the use of a low dose of an inhalational anesthetic agent combined with the infusion of a potent opioid (sufentanil or remifentanil) [9]. When inhalational anesthetic agents are used, it is generally recommended to maintain end-tidal concentrations levels at or below 0.5 MAC (minimum alveolar concentration) to allow for adequate MEP monitoring. These agents provide an effective intraoperative anesthetic while allowing for neurophysiological monitoring and providing a rapid emergence at the conclusion of the case.

In summary, we present the perioperative management of an adolescent with MFS for spinal fusion. As the primary defect affects fibrillin-1, a matrix glycoprotein essential for the formation and repair of connective tissue, multisystem involvement is present. Progressive skeletal deformities may require orthopedic procedures such as spinal fusion. Most importantly, the cardiovascular system may be involved with valvular regurgitation, aortic root dilation, and arrhythmias. Additional comorbid concerns include airway involvement (difficult intubation, sleep disordered breathing), respiratory involvement (risk of barotrauma), and ocular involvement. The preoperative evaluation is essential to identify anatomical and end-organ effects of MFS, as well as implications of therapeutic interventions, such as long-term anticoagulation.

\section{Grant Support}

Not applicable.

\section{Conflict of Interest}

The authors do not have conflict of interest.

\section{References}

1. Hecht F, Beals RK. "New" syndrome of congenital contractural arachnodactyly originally described by Marfan in 1896. Pediatrics. 1972;49(4):574-579.

2. Judge DP, Dietz HC. Marfan's syndrome. Lancet. 2005;366(9501):1965-1976.

3. Loeys BL, Dietz HC, Braverman AC, Callewaert BL, De Backer J, Devereux RB, Hilhorst-Hofstee Y, et al. The revised Ghent nosology for the Marfan syndrome. J Med Genet. 2010;47(7):476-485.

4. Faivre L, Collod-Beroud G, Loeys BL, Child A, Binquet C, Gautier E, Callewaert B, et al. Effect of mutation type and location on clinical outcome in 1,013 probands with Marfan syndrome or related phenotypes and FBN1 mutations: an international study. Am J Hum Genet. 2007;81(3):454-466.

5. Dietz HC, Cutting GR, Pyeritz RE, Maslen CL, Sakai LY, Corson GM, Puffenberger EG, et al. Marfan syndrome caused by a recurrent de novo missense mutation in the fibrillin gene. Nature. 1991;352(6333):337-339.
6. Matt P, Schoenhoff F, Habashi J, Holm T, Van Erp C, Loch D, Carlson OD, et al. Circulating transforming growth factor-beta in Marfan syndrome. Circulation. 2009;120(6):526-532.

7. Benke K, Agg B, Szilveszter B, Tarr F, Nagy ZB, Polos $\mathrm{M}$, Daroczi L, et al. The role of transforming growth factor-beta in Marfan syndrome. Cardiol J. 2013;20(3):227234.

8. Castellano JM, Silvay G, Castillo JG. Marfan syndrome: clinical, surgical, and anesthetic considerations. Semin Cardiothorac Vasc Anesth. 2014;18(3):260-271.

9. Martin DP, Bhalla T, Thung A, Rice J, Beebe A, Samora W, Klamar J, et al. A preliminary study of volatile agents or total intravenous anesthesia for neurophysiological monitoring during posterior spinal fusion in adolescents with idiopathic scoliosis. Spine (Phila Pa 1976). 2014;39(22):E1318-1324.

10. Dziewit JA, Wei H. Supraglottic jet ventilation assists intubation in a Marfan's syndrome patient with a difficult airway. J Clin Anesth. 2011;23(5):407-409.

11. Aiello G, Metcalf I. Anaesthetic implications of temporomandibular joint disease. Can J Anaesth. 1992;39(6):610616.

12. Bauss O, Sadat-Khonsari R, Fenske C, Engelke W, Schwestka-Polly R. Temporomandibular joint dysfunction in Marfan syndrome. Oral Surg Oral Med Oral Pathol Oral Radiol Endod. 2004;97(5):592-598.

13. Engelhardt T, Weiss M. A child with a difficult airway: what do I do next? Curr Opin Anaesthesiol. 2012;25(3):326-332.

14. Bryant J, Krishna SG, Tobias JD. The difficult airway in pediatrics. Advances in Anesthesia. 2013;31(1):31-60.

15. Cistulli PA, Richards GN, Palmisano RG, Unger G, Berthon-Jones M, Sullivan CE. Influence of maxillary constriction on nasal resistance and sleep apnea severity in patients with Marfan's syndrome. Chest. 1996;110(5):1184-1188.

16. Cistulli PA, Sullivan CE. Sleep apnea in Marfan's syndrome. Increased upper airway collapsibility during sleep. Chest. 1995;108(3):631-635.

17. Memtsoudis SG, Cozowicz C, Nagappa M, Wong J, Joshi GP, Wong DT, Doufas AG, et al. Society of anesthesia and sleep medicine guideline on intraoperative management of adult patients with obstructive sleep apnea. Anesth Analg. 2018;127(4):967-987.

18. Karpman C, Aughenbaugh GL, Ryu JH. Pneumothorax and bullae in Marfan syndrome. Respiration. 2011;82(3):219-224.

19. Koumbourlis AC. Scoliosis and the respiratory system. Paediatr Respir Rev. 2006;7(2):152-160.

20. Vedantam R, Lenke LG, Bridwell KH, Haas J, Linville DA. A prospective evaluation of pulmonary function in patients with adolescent idiopathic scoliosis relative to the surgical approach used for spinal arthrodesis. Spine (Phila Pa 1976). 2000;25(1):82-90.

21. Streeten EA, Murphy EA, Pyeritz RE. Pulmonary function in the Marfan syndrome. Chest. 1987;91(3):408-412.

22. Almenrader N, Patel D. Spinal fusion surgery in children with non-idiopathic scoliosis: is there a need for routine 
postoperative ventilation? Br J Anaesth. 2006;97(6):851857.

23. Jaber S, Michelet P, Chanques G. Role of non-invasive ventilation (NIV) in the perioperative period. Best Pract Res Clin Anaesthesiol. 2010;24(2):253-265.

24. Pelosi P, Jaber S. Noninvasive respiratory support in the perioperative period. Curr Opin Anaesthesiol. 2010;23(2):233-238.

25. Judge DP, Rouf R, Habashi J, Dietz HC. Mitral valve disease in Marfan syndrome and related disorders. J Cardiovasc Transl Res. 2011;4(6):741-747.

26. Rybczynski M, Mir TS, Sheikhzadeh S, Bernhardt AM, Schad C, Treede H, Veldhoen S, et al. Frequency and agerelated course of mitral valve dysfunction in the Marfan syndrome. Am J Cardiol. 2010;106(7):1048-1053.

27. Tsang VT, Pawade A, Karl TR, Mee RB. Surgical management of Marfan syndrome in children. J Card Surg. 1994;9(1):50-54.

28. Salim MA, Alpert BS, Ward JC, Pyeritz RE. Effect of beta-adrenergic blockade on aortic root rate of dilation in the Marfan syndrome. Am J Cardiol. 1994;74(6):629633.

29. Shores J, Berger KR, Murphy EA, Pyeritz RE. Progression of aortic dilatation and the benefit of long-term beta-adrenergic blockade in Marfan's syndrome. N Engl J Med. 1994;330(19):1335-1341.

30. Ammash NM, Sundt TM, Connolly HM. Marfan syndrome-diagnosis and management. Curr Probl Cardiol. 2008;33(1):7-39.

31. Lacro RV, Dietz HC, Sleeper LA, Yetman AT, Bradley TJ, Colan SD, Pearson GD, et al. Atenolol versus losartan in children and young adults with Marfan's syndrome. N Engl J Med. 2014;371(22):2061-2071.

32. Kluin J, Koolbergen DR, Sojak V, Hazekamp MG. Valvesparing root replacement in children. Eur J Cardiothorac Surg. 2016;50(3):476-481.

33. Martens A, Beckmann E, Kaufeld T, Fleissner F, Neuser J, Korte W, Merz C, et al. Valve-sparing aortic root replacement (David I procedure) in Marfan disease: single-centre 20-year experience in more than 100 patients.
Eur J Cardiothorac Surg. 2018.

34. Tweddell JS, Earing MG, Bartz PJ, Dunham-Ingles JL, Woods RK, Mitchell ME. Valve-sparing aortic root reconstruction in children, teenagers, and young adults. Ann Thorac Surg. 2012;94(2):587-590; discussion 590581.

35. Nishimura RA, Otto CM, Bonow RO, Carabello BA, Erwin JP, 3rd, Fleisher LA, Jneid H, et al. 2017 AHA/ACC focused update of the 2014 AHA/ACC guideline for the management of patients with valvular heart disease: a report of the American College of Cardiology/American Heart Association task force on clinical practice guidelines. Circulation. 2017;135(25):e1159-e1195.

36. Yetman AT. Cardiovascular pharmacotherapy in patients with Marfan syndrome. Am J Cardiovasc Drugs. 2007;7(2):117-126.

37. Savolainen A, Kupari M, Toivonen L, Kaitila I, Viitasalo M. Abnormal ambulatory electrocardiographic findings in patients with the Marfan syndrome. J Intern Med. 1997;241(3):221-226.

38. Kitaba A, Martin DP, Gopalakrishnan S, Tobias JD. Perioperative visual loss after nonocular surgery. J Anesth. 2013;27(6):919-926.

39. Ho VT, Newman NJ, Song S, Ksiazek S, Roth S. Ischemic optic neuropathy following spine surgery. J Neurosurg Anesthesiol. 2005;17(1):38-44.

40. American Society of Anesthesiologists Task Force on Perioperative Visual L. Practice advisory for perioperative visual loss associated with spine surgery: an updated report by the American Society of Anesthesiologists Task Force on Perioperative Visual Loss. Anesthesiology. 2012;116(2):274-285.

41. Sloan TB, Heyer EJ. Anesthesia for intraoperative neurophysiologic monitoring of the spinal cord. J Clin Neurophysiol. 2002;19(5):430-443.

42. Balvin MJ, Song KM, Slimp JC. Effects of anesthetic regimens and other confounding factors affecting the interpretation of motor evoked potentials during pediatric spine surgery. Am J Electroneurodiagnostic Technol. 2010;50(3):219-244. 